\title{
Guías de práctica clínica sobre el tratamiento del traumatismo craneoencefálico leve en adultos
}

Grupo de estudio del traumatismo craneoencefálico de la sociedad italiana de neurocirugía

Journal of Neurosurgical Sciences 40:11-15, 1996.

Refrendado por los miembros del GEN (Grupo Español de Neurotraumatología)*

El Grupo de Estudio del Traumatismo Craneoencefálico de la Sociedad Italiana de Neurocirugía establece las siguientes guías de práctica clínica para el tratamiento de los pacientes con traumatismo craneoencefálico leve. En este grupo se incluyen los pacientes orientados en tiempo, espacio y persona (GCS 15) y los pacientes desorientados (GCS 14). La presencia de déficits neurológicos focales, el traumatismo abierto y un GCS $\leq 13$ constituyen criterios de exclusión. Se identificaron seis categorías de factores de riesgo relevantes para el curso clínico (coagulopatías, enolismo, abuso de drogas, epilepsia, antecedentes neuro-quirúrgicos y pacientes ancianos incapacitados). Se distinguieron tres grupos de pacientes. Los pacientes del Grupo 0 (GCS 15, sin pérdida de conciencia, amnesia, cefalea difusa, vómitos) que pudieron darse de alta del Departamento de Urgencias tras un periodo de observación de seis horas con un informe de asistencia. Los pacientes del Grupo 1 (GCS 15 , con pérdida de conciencia $y / 0$ amnesia y/o cefalea difusa y/o vómitos) requieren observación clínica $(\geq 6$ horas) y valoración neurorradiológica. Según la disponibilidad del hospital, se realizará radiología simple de cráneo o tomografía axial computarizada (TAC). Ante una fractura de cráneo, es obligada la TAC. En caso de lesiones intracraneales, se consultará al neurocirujano. En ausencia de fracturas craneales o de lesiones intracraneales, se ingresa al paciente para observación (( $\geq 24$ horas). Los pacientes del Grupo 0 y del Grupo 1 con un factor de riesgo $(R)$ se ingresan ( $\geq 24$ horas) para realizar la TAC. En pacientes con coagulopatías o en tratamiento con anticoagulantes, deberá repetirse la TAC antes del alta incluso en ausencia de lesión intracraneal en la TAC inicial. En pacientes del Grupo 2 (GCS 14), se realiza TAC en todos los casos, independientemente de la existencia de factor de riesgo. [ $\mathrm{J}$ Neurosurg Sci 1996;40:11-5]

PALABRAS CLAVE: Traumatismo craneoencefálico. Neurocirugía
La gran mayoría de pacientes con traumatismo craneoencefálico (TCE) atendidos en Urgencias de nuestros hospitales están completamente conscientes en el examen inicial. El tratamiento de tales TCE leves se centra en el riesgo de desarrollar un hematoma intracraneal traumático y en la necesidad de detectar y evacuar el coágulo precozmente ${ }^{1}$. Este hecho es excepcional (del 1\% al 3\% de los pacientes ingresados con diagnóstico de TCE leve ${ }^{1-5}$. Puesto que en Italia la frecuentación hospitalaria por cada 100.000 habitantes es de 300-400 casos/año tras $\mathrm{TCE}^{6}$, la búsqueda de un paciente con hematoma supondría un esfuerzo organizativo considerable.

Además, a diferencia de EEUU y al igual que en muchos otros países europeos, se dispone de unidades neuroquirúrgicas en unos pocos hospitales. Por tanto, con relativa frecuencia, el tratamiento de los pacientes con TCE leve no se realiza en dichos centros. Cualquier protocolo sobre TCE leve debe tener en cuenta los recursos disponibles no únicamente en los pocos hospitales que disponen de unidades neuroquirúrgicas sino, más importante aún, de todos los hospitales en donde este tipo de pacientes serán tratados. Unicamente tenemos conocimiento de un informe publicado en 1984 por un grupo de neurocirujanos británicos ${ }^{7}$ que pretendió establecer guías de práctica clínica nacionales (Gran Bretaña) para pacientes con TCE leve.

El objetivo de este artículo es establecer guías de práctica clínica para el tratamiento del TCE leve para la utilización en la práctica clínica de nuestro país (Italia).

\footnotetext{
* El Grupo Español de Neurotraumatologia (GEN) está formado por los siguientes miembros: Alted, E., (Cuidados Intensivos), Díez, R., (Neurocirugía), Domínguez, J.M., (Cuidados Intensivos), García, R., (Anestesiología y Reanimación), Garibi, J., (Neurocirugía), Gómez, P.A., (Neurocirugía), Gracia, R.M., (Cuidados Intensivos), Jiménez, R., (Urgencias y Emergencias), Millán, J.M., (Neurorradiología), Murillo, F., (Cuidados Intensivos), Puig, A., (Anestesiología y Reanimación), Sahuquillo, J., (Neurocirugía), Soto, J.M., (Urgencias y Emergencias).
} 


\section{Material y métodos}

Los pacientes son atendidos en Urgencias por médicos de urgencias o por neurólogos, neurocirujanos o cirujanos generales. La evaluación de los pacientes se realiza a través de un examen neurológico, la puntuación de la Escala de Coma de Glasgow (GCS) y la existencia de pérdida de conciencia, amnesia, cefalea, vómitos y vértigo.

Los pacientes con déficits neurológicos focales y traumatismo abierto son excluidos del grupo de TCE leve, ya que el riesgo de hematoma intracraneal es mayor ${ }^{8}$.

También se excluyeron los pacientes con puntuación GCS de 3 a 8 (TCE grave) y de 9 a 13 (TCE moderado). La puntuación GCS de 13 se incluye en el grupo de TCE moderado (a diferencia de la clasificación original de Rimel y col. ${ }^{9}$, ya que el riesgo de deterioro es similar ${ }^{10}$. Por tanto, al ingreso, estos pacientes están orientados temporoespacialmente y no presentan déficits neurológicos $(\mathrm{GCS}=15)$ o están desorientados con ausencia de déficits neurológicos (GCS 14).

Se identificaron factores de riesgo relevantes en el curso clínico de los pacientes. Entre estos factores se cuentan las coagulopatías $^{11}$, enolismo ${ }^{12}$, abuso de drogas, epilepsia, antecedentes neuroquirúrgicos y pacientes ancianos incapacitados $^{13}$.

Siempre que exista uno o más de estos factores, se añade $\mathrm{R}$ a la clasificación.

\section{Indicaciones de tratamiento}

En la Figura 1, se presenta el algoritmo para los pacientes del grupo 0. Estos pacientes con traumatismo francamente leve pueden ser dados de alta desde Urgencias tras un período mínimo de observación de 6 horas con un informe de asistencia. En estos casos no se requiere examen radiológico ${ }^{7}$.

En las Figuras 2 y 3, se presenta el algoritmo para los pacientes del grupo 1. Estos pacientes son los más difíciles de evaluar. Las indicaciones que aparecen en la literatura para tales casos son radiología simple ${ }^{7}$, TAC en los casos con deterioro clínico ${ }^{2}$, TAC en todo paciente adulto con fractura de cráneo $^{14}$, TAC en todo paciente de cualquier edad con fractura de cráneo ${ }^{1}$ y TAC en todos los pacientes con TCE leve.'

Obviamente, las indicaciones de la TAC depende de los recursos disponibles; en toda Italia consideramos que no hay suficientes escáneres disponibles 24 horas al día para estudiar toda la población por este problema clínico. Aún en el caso de que fuera posible realizar una TAC en tales pacientes en unas pocas áreas y centros, se haría necesaria una selección en el contexto de un protocolo nacional.

En un paciente con TCE leve, la incidencia de deterioro clínico es del $0,2 \%$ al $0,7 \%$ en caso de ausencia de fractura de cráneo y del 3,2\% al $10 \%$ en adultos con fractura ${ }^{2-5}$. Uno de los objetivos de las guías de práctica clínica no es identificar una lesión intracraneal sino identificar pacientes portadores de una "masa intracraneal relevante"16, principalmente hematomas epidurales antes del deterioro clínico. Por tanto, es indudable el valor de la fractura de cráneo para identificar una población de mayor riesgo de hematoma. En un amplio ensayo clínico multicéntrico sobre TCE leve (realizado en 2766 pacientes), Sackford y col. ${ }^{16}$ demostraron que la existencia de fractura de cráneo triplicaba el riesgo de lesión intracraneal "relevante". Además, un estudio multicéntrico italiano sobre hematoma epidural asintomático demostró la existencia de una fractura de cráneo en el $89 \%$ de los casos en adultos ${ }^{17}$. No se dispone de indicadores clínicos de hematoma epidural en fase asintomática $^{18,19}$.

La decisión es realizar la TAC de rutina a todo paciente ${ }^{19} \mathrm{o}$ únicamente a los pacientes adultos con fractura de cráneo ${ }^{1,14}$. Incluso cuando es posible realizar la TAC, una prueba normal en las primeras horas del traumatismo no descarta, cuando hay fractura de cráneo, la aparición de un hematoma epidural tardío ( 2 casos de 31 pacientes) ${ }^{20}$. En el tratamiento del paciente es relevante saber si existe fractura de cráneo ${ }^{16}$.

Por tanto, los pacientes del Grupo 1 (Fig. 3) requieren observación clínica y valoración neurorradiológica. Según la disponibilidad del hospital, en un período mínimo de 6 horas de observación, se realizará radiología de cráneo o TAC. Cuando exista fractura de cráneo, es obligado practicar TAC. En caso de lesión intracraneal, se consultará al neurocirujano. En ausencia de fractura de cráneo o de lesiones intracraneales, el paciente se ingresará para observación mínima de 24 horas.

En las Figuras 4 y 5 aparece el tratamiento de los pacientes con desorientación persistente (GCS 14), grupo 2. Estos pacientes representan un pequeño subgrupo (menos del 7\% de los casos) $)^{18,21}$ y presentan un mayor riesgo de hematoma intracraneal. Por tanto, en estos casos se realizará TAC independientemente de la existencia de factores de riesgo.

Los pacientes del grupo $0 \mathrm{y}$ del grupo 1 con un factor de riesgo, R (Fig. 6) se ingresan en el hospital durante al menos 24 horas y se realiza TAC. En pacientes con coagulopatías o en tratamiento con anticoagulantes, deberá repetirse la TAC antes del alta, incluso en ausencia de lesión intracraneal en la TAC previa.

En estos casos es frecuente el hematoma intraparenquimatoso tardío, especialmente en los ancianos ${ }^{22}$.

Siempre que exista una lesión intracraneal postraumática, se consultará un neurocirujano. Las lesiones extracerebrales pequeñas pueden evolucionar en pocas horas ${ }^{23} \mathrm{y}$, en estos casos, la observación clínica puede ser más segura en la planta de neurocirugía. 


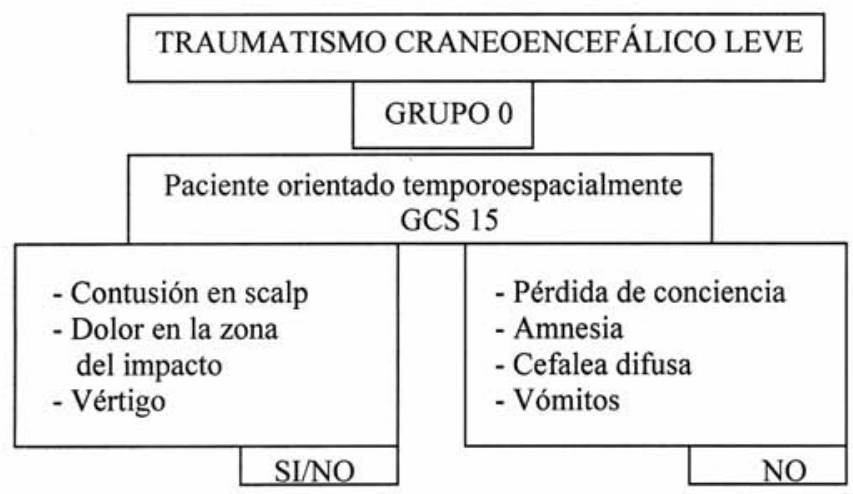

Fig. 1 .- Definición de pacientes con traumatismo craneoencefálico leve: Grupo 0

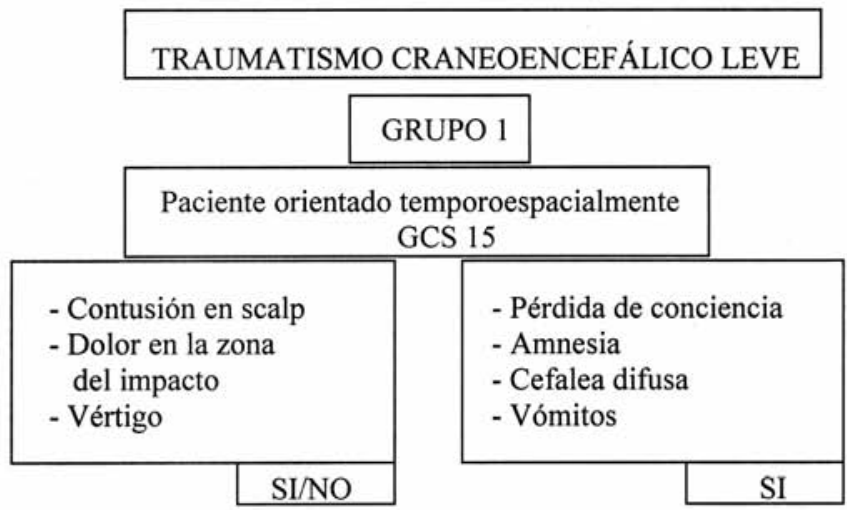

Fig. 2 .- Definición de pacientes con traumatismo craneoencefálico leve: Grupo 1

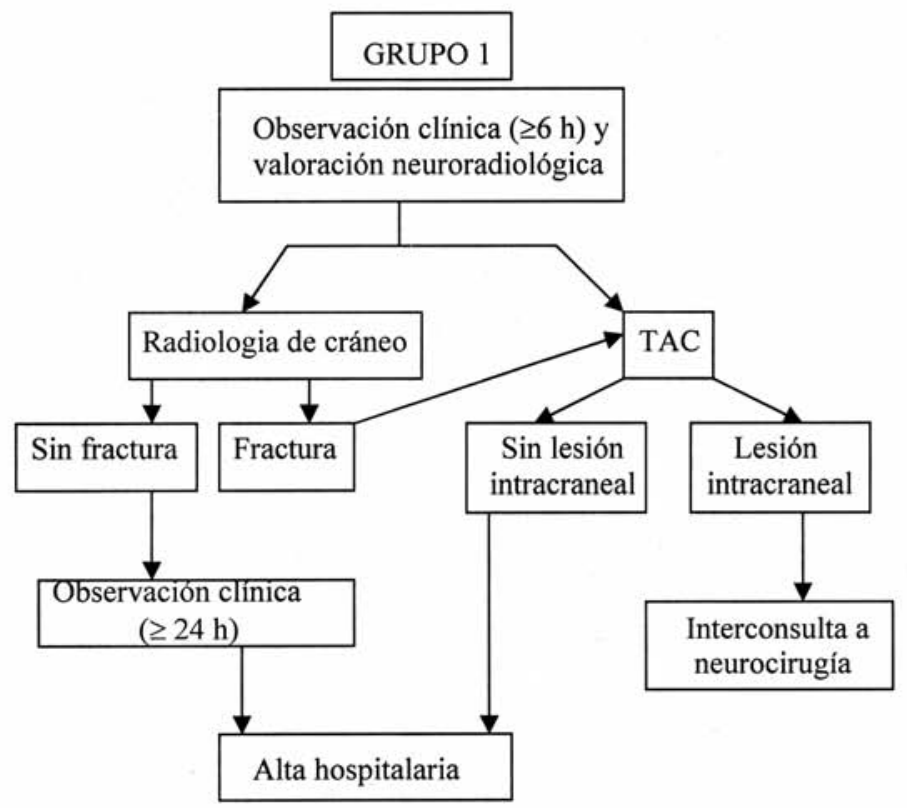

Fig. 3 .- Algoritmo de tratamiento de los pacientes con traumatismo craneoencefálico leve: Grupo 1 


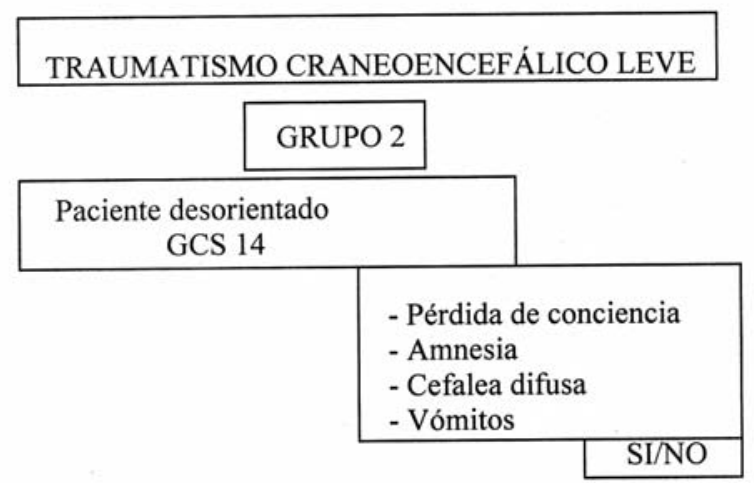

Fig. 4 -- Definición de pacientes con traumatismo craneoencefálico leve: Grupo 2

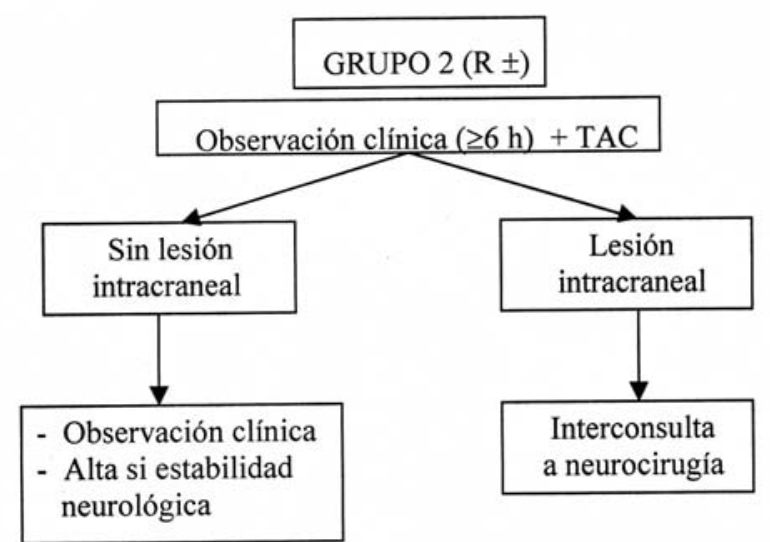

Fig. 5 - Algoritmo de tratamiento de los pacientes con traumatismo craneoencefálico leve: Grupo 2, sin o con un factor de riesgo $(\mathrm{R} \pm)$.

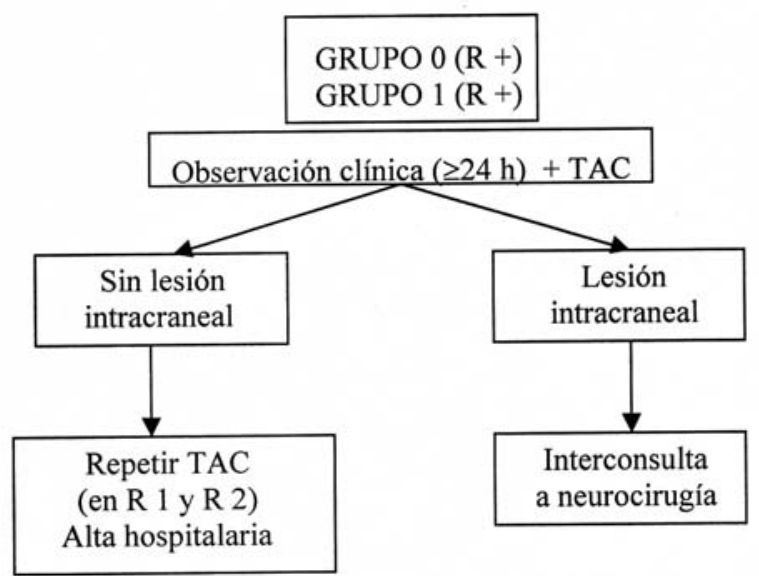

Fig. 6 -- Algoritmo de tratamiento de los pacientes con traumatismo craneoencefálico leve: Grupo 0 y Grupo 1 , sin o con un factor de riesgo $(\mathrm{R} \pm)$. 


\section{Conclusiones}

El objetivo principal de estas guías de práctica para el tratamiento inicial de adultos con TCE en Italia es reunir los criterios globales que permitan identificar pacientes con TCE leve con riesgo de desarrollar hematoma intracraneal.

Las guías de práctica clínica incluyen la evaluación de la historia clínica traumatológica, los exámenes clínicos y neurorradiológicos y la identificación de factores de riesgo. Varios algoritmos muestran los criterios de valoración neurorradiológica, ingreso y alta hospitalarias, interconsulta a neurocirugía según el riesgo de lesión intracraneal en diferentes grupos clínicos. Como el tratamiento del TCE leve debe organizarse en todo el país, las guías de práctica clínica se dirigirán a todos los servicios de Urgencias y no únicamente a unos pocos centros especializados. Por consiguiente, las guías de práctica clínica deben tener en cuenta los recursos disponibles en todos los centros del país.

\section{Addendum}

De acuerdo con los criterios de un Grupo de Neurocirujanos del Reino Unido, los pacientes con herida de cuero cabelludo deberían ser incluidos en el Grupo 1.

\section{Título de la referencia}

\section{Guidelines for the initial management of head injuries}

\section{Recommendations from the Society of British Neurolo- gical Surgeons}

Members of the working party of the society of british neurological surgeons: J. Bartlett, R., Kett-White, A.D., Mendelow, J.D., Miller, J., Pickard \& G. Teasdale.

British Journal of Neurosurgery 1998;12(4):349-352.

\section{Bibliografía}

1. Teasdale, G.M., Murray, G., Anderson, E. et al.: Risks of acute intracranial hematoma in children and adults: implications for managing head injuries. Br Med J 1990; 300: 363-367.

2. Dacey, R.G., Wayne, A.M., Rimel, R.W., Wim, R., Jane, J.A.: Neurosurgical complications after apparently minor head injury: assessment of risk in a series of 160 patients. J Neurosuerg 1986; 65: 203-210.

3. Edna, T.H.: Acute intracranial haematoma and skull fracture. Acta Chir Scand 19832; 149: 449-451.

4. Mendelow, A.D., Teasdale, G., Jennet, B., Bryden, J., Hasset, C., Murray, G.: Risks of intracranial hematoma in head injured adults. Br Med J 1983; 287:1173-1176.

5. Miller, J.D.: Minor, moderate and severe head injury.
Neurosurg Rev 1986; 9:135-139.

6. Servadei, F., Ciucci, G., Piazza, G. et al.: A prospective clinical and epidemiological study of head injures in Northern Italy: the Comune of Ravenna. Ital J Neurol Sci 1988; 9: 449-457.

7. Briggs, M., Clarke, P., Crokard, A.: British Neurosurgeons Group. Guidelines for initial management after head injuries in adults. Br Med J 1984; 288: 983-985.

8. Miller, J.D., Murray, L.S., Teasdale, G.M.: Development of a traumatic intracranial hematoma after a minor head injury. Neurosurgey 1990; 27: 669-673.

9. Rimel, E.W., Giordani, B., Barth, J.T., Boll, T.J., Jane, J.A.: Disability caused by minor head injury. Neurosurgery 1981; 9: 221-228.

10. Stein, C.C., Ross, S.E.: Moderate head injury: a guide to initial management. J Neurosurgery 1992; 77: 562-564.

11. Olson, J.D., Kaufman, H.H., Moake, J. et al.: The incidence and significance of hemostatic abnormalities in patients with head injures. Neurosurgery 1989; 24: 825-832.

12. Nath, F.P., Beastal, G., Teasdale, G.M.: Alcohol and traumatic brain damage. Injury 1986; 17: 150-153.

13. Wilson, J.A., Pentland, B., Currie, C.T., Miller, J.D.: The functional effects of head injury in elderly. Brain Injury 1987; 1: 183-188.

14. Servadei, F., Ciucci, G., Morichetti, A. et al.: Skull fracture as a factor of increased risk in minor head injuries. Surg Neurol 1988; 30: 364-369.

15. Stein, S.C., Ross, S.E.: Mild head injury: A plea for routine early CT scanning. J Trauma 1992; 33: 11-13.

16. Schackford, S.R., Wald, S.L., Ross, S.E. et al.: The clinical utility of computed Tomographic scanning and neurological examination in the management of patients with minor head injury. J Trauma 1992; 33:22-29.

17. Servadei, F., Faccani, G., Rocella, P. et al.: Asymptomatic extradural haematomas. Results of a multicenter study of 158 cases in minor head injury. Acta Neurochir 1989; 96: 39-45.

18. Servadei, F., Ciucci, G., Loroni, L., Cuscini, M., Piola, C., Arista, A.: The diagnosis and management of minor head injury: a regional multicenter approach in Italy. J Trauma (in press), 1995.

19. Stein, S.C., Spettel, C., Young, G., Ross, S.E.: Limitations of neurological assessment in mild head injury. Brain Injury 1993; 7: 425-430.

20. Poon, W.S., Poon, C.Y.S., Li, A.K.C.: Traumatic extradural hematoma of delayed onset is not a rarity. Neurosurgery 1992; 30:681-686.

21. Miller, J.D., Jones, P.A.: The work of a regional head injury service. Lancet 1985; ii:1141-1144.

22. Stein, S.C., Young, G.S., Talucci, R.C., Greenbaum, B.H., Ross, S.E.: Delayed brain injury following head trauma: signicance of coagulopathy. Neurosurgery 1992; 30:160-165.

23.Smith, H.K., Miller, J.D.: The danger of an ultra early computed tomographic scan in a patient with an evolving intracranial hematoma. Neurosurgey 1991; 29:258-60. 